\title{
Characterization, Tissue Expression, and Imprinting Analysis of the Porcine CDKN1C and NAP1L4 Genes
}

\author{
Shun Li, ${ }^{1}$ Juan Li, ${ }^{1}$ Jiawei Tian, ${ }^{1}$ Ranran Dong, ${ }^{1}$ Jin Wei, ${ }^{1}$ Xiaoyan Qiu,, ${ }^{2}$ and Caode Jiang ${ }^{2}$ \\ ${ }^{1}$ School of Life Science, Southwest University, Chongqing 400715, China \\ ${ }^{2}$ College of Animal Science and Technology, Southwest University, Chongqing 400715, China
}

Correspondence should be addressed to Caode Jiang, caodejiang@hotmail.com

Received 4 August 2011; Revised 25 October 2011; Accepted 15 November 2011

Academic Editor: Andre Van Wijnen

Copyright () 2012 Shun Li et al. This is an open access article distributed under the Creative Commons Attribution License, which permits unrestricted use, distribution, and reproduction in any medium, provided the original work is properly cited.

\begin{abstract}
CDKN1C and NAP1L4 in human CDKN1C/KCNQ1OT1 imprinted domain are two key candidate genes responsible for BWS (Beckwith-Wiedemann syndrome) and cancer. In order to increase understanding of these genes in pigs, their cDNAs are characterized in this paper. By the IMpRH panel, porcine CDKN1C and NAP1L4 genes were assigned to porcine chromosome 2, closely linked with IMpRH06175 and with LOD of 15.78 and 17.94, respectively. By real-time quantitative RT-PCR and polymorphism-based method, tissue and allelic expression of both genes were determined using F1 pigs of Rongchang and Landrace reciprocal crosses. The transcription levels of porcine CDKN1C and NAP1L4 were significantly higher in placenta than in other neonatal tissues $(P<0.01)$ although both genes showed the highest expression levels in the lung and kidney of onemonth pigs $(P<0.01)$. Imprinting analysis demonstrated that in pigs, CDKN1C was maternally expressed in neonatal heart, tongue, bladder, ovary, spleen, liver, skeletal muscle, stomach, small intestine, and placenta and biallelically expressed in lung and kidney, while NAP1L4 was biallelically expressed in the 12 neonatal tissues examined. It is concluded that imprinting of CDKN1C is conservative in mammals but has tissue specificity in pigs, and imprinting of NAP1L4 is controversial in mammalian species.
\end{abstract}

\section{Introduction}

Genomic imprinting, in which the monoallelic expression of certain genes depends on the parent of origin due to epigenetic regulation in somatic cells, is vital for fetal growth and development, function of the placenta, and postnatal behaviour in mammals. Many researches have reported that disruption of genomic imprinting is responsible for congenital disorders, cancers, and other types of diseases such as BWS (Beckwith-Wiedemann syndrome) and PWS (PraderWilli syndrome) [1-4]. Currently, about 2114 imprinted transcripts and 600 potentially imprinted genes have been estimated in the genome of mice $[5,6]$, of which about 160 imprinted genes have been described (http://igc.otago.ac.nz/ home.html), but only several genes have been reported to be imprinted in pigs. Therefore, it is necessary to characterize more imprinted genes in the pig to offer new data and insights to the comparative genome research as well as porcine functional genomics.

Most imprinted genes aggregate within the genome and gene order and imprinting in each imprinted domain show considerable similarity between mouse and human $[7,8]$. It has been reported that CDKN1C and KCNQ1OT1 reside in an imprinted cluster on human 11p15.5, syntenic to mouse distal chromosome 7 . Genes in this region include OSBPL5, CARS, NAP1L4, PHLDA2, SLC22A18, SLC22A18AS, CDKN1C, KCNQ1DN, KCNQ1OT1, KCNQ1, TSSC4, CD81, and ASCL2 [8]. Imprinting disruption of the CDKN1C/KCNQ1OT1 domain is involved in the development of both BWS and cancer [8].

CDKN1C (cyclin-dependant kinase inhibitor) and NAP1L4 (nucleosome assembly protein 1-like 4) are two of particular interest genes within the CDKN1C/KCNQ1OT1 imprinted cluster in human and mouse. The CDKN1C, also known as $\mathrm{p} 57^{\mathrm{Kip} 2}$, encodes a cyclin-dependent kinase inhibitor (CDKI) that belongs to the CIP/KIP family, and it is a putative tumor suppressor gene $[9,10]$. Mutations in CDKN1C have been identified in approximately 5-20\% of patients with BWS [11, 12], and mice with maternally inherited deletion of CDKN1C showed some features of BWS [13], while in sporadic cancers and embryonal tumors, 
mutations of CDKN1C are very rare [14-18]. NAP1L4, also called NAP-2, codes for a protein with homology to NAPs involved in regulating gene expression by delivering histones from the cytoplasm to the chromatin assembly machinery [19]. NAP1L4 associates with the processes of restraining fetal growth and causing fetal lethality [20]. These results suggest that CDKN1C and NAP1L4 are two of the important genes responsible for the development of BWS and cancer [8].

At present, there are a lot of reports about the imprinting status of CDKN1C and NAP1L4 both in human and mouse. CDKN1C is expressed from the maternal allele, while NAP1L4 is biallelically expressed in all the tissues except maternal expression in mouse placenta [21]. In the pig, CDKN1C is expected of maternal expression [22]. Despite of these advances, data on porcine CDKN1C and NAP1L4 are limited. Hence, tissue expression patterns and imprinting status of porcine CDKN1C and NAP1L4 were investigated in this work. This study will contribute to increasing the understanding of functional importance of CDKN1C and NAP1L4 in pigs.

\section{Materials and Methods}

2.1. Tissue Samples and cDNA Preparations. Reciprocal crossings were performed using 8 Landrace (LD) and 8 indigenous China breed Rongchang (RC) pigs (4 boars and 4 sows of each breed). These boars and sows have no common grandparents. Tissues including heart, stomach, skeletal muscle, kidney, lung, liver, small intestine, bladder, tongue, spleen, fat, ovary and placenta were collected from $23 \mathrm{~F} 1$ pigs, which included 10 neonatal pigs within $24 \mathrm{~h}$ after birth (5 pigs from three parent pairs of $\mathrm{LD} \times \mathrm{RC}$ and 5 pigs from three parent pairs of RC $\times \mathrm{LD}$ ) as well as 7 and 6 onemonth pigs from four parent pairs of $\mathrm{LD} \times \mathrm{RC}$ and $\mathrm{RC} \times \mathrm{LD}$, respectively. Ear tissues of parental pigs for genomic DNA isolation and genotyping of CDKN1C and NAP1L4 were also collected. Tissue samples were stored at $-80^{\circ} \mathrm{C}$ until use.

Total RNA was isolated from frozen tissues using TRIzol reagent (Invitrogen, San Diego, USA) and treated with DNase I (TaKaRa, Japan). Reverse transcription was carried out using AMV reverse transcriptase XL (TaKaRa, Japan) and oligo(dT) primer at $42^{\circ} \mathrm{C}$ for $50 \mathrm{~min}, 70^{\circ} \mathrm{C}$ for $15 \mathrm{~min}$. Both reactants with and without reverse transcriptase were used as positive and negative samples, respectively, for gene cloning and imprinting analysis.

2.2. Molecular Cloning of CDKN1C and NAP1L4. The cDNA sequences of human genes CDKN1C (NM_000076) and NAP1L4 (NM_005969) were used to search for available expressed sequence tags (ESTs) in the pig ESTs database using BLASTn program (http://www.ncbi.nlm.nih.gov/BLAST/). The homologous sequences, sharing greater than $80 \%$ identity and match of at least $100 \mathrm{bp}$, were assembled into ESTs contigs for primer design. Primer pair CDF1/R1 was used for CDKN1C, while NAF1/R1 for NAP1L4 (Table 1). To obtain full-length cDNA of the two genes, $5^{\prime}$ - and $3^{\prime}$-RACE were performed with SMART RACE cDNA Amplification Kit
(Clontech, USA) and the RNA LA PCR Kit (TaKaRa, Japan), respectively, according to the supplier's instructions. For 5' RACE, sequence-specific primers $5^{\prime}$ CDGSP1 and 5'CDGSP2 (nested) were designed for CDKN1C, while $5^{\prime}$ NAGSP1 and $5^{\prime}$ NAGSP2 (nested) for NAP1L4. For 3'-RACE, sequencespecific primers, $3^{\prime} \mathrm{NAGSP} 1$ and $3^{\prime} \mathrm{NAGSP} 2$ nested were designed for NAP1L4.

Thermocycling conditions of PCR reactions were initial denaturation at $94^{\circ} \mathrm{C}$ for $5 \mathrm{~min}$, followed by 35 cycles of denaturation at $94^{\circ} \mathrm{C}$ for $30 \mathrm{~s}$, annealing at $53-60^{\circ} \mathrm{C}$ for $30 \mathrm{~s}$ (Table 1), and extension at $72^{\circ} \mathrm{C}$ for $30 \mathrm{~s}$, with a final extension at $72^{\circ} \mathrm{C}$ for $10 \mathrm{~min}$. PCR products were then sequenced at commercial service (Invitrogen, Shanghai, China).

2.3. Radiation Hybrid (RH) Mapping. To obtain intron sequence for RH mapping, alignment of porcine CDKN1C and NAP1L4 with human corresponding genes (NM_000076, NM_005969) was performed. According to the consensus sequences, primers INF1/R1 and INF2/R2 (Table 1) were designed for amplification of CDKN1C intron 2 and NAP1L4 intron 3. Primer pairs MF1/R1 and MF2/R2 (Table 1) based on the introns above were used for PCR typing of the porcine radiation hybrid (IMpRH) panel, which were kindly provided by INRA (France) [23]. PCR was performed in duplicate in a total volume of $10 \mu \mathrm{L}$, containing $25 \mathrm{ng} \mathrm{IMpRH}$ DNA samples, $0.3 \mu \mathrm{M}$ of each primer, $75 \mu \mathrm{M}$ of each dNTP, $1.5 \mathrm{mM} \mathrm{MgCl}_{2}$, and $1 \mathrm{U}$ Taq DNA polymerase (TaKaRa, Japan). The PCR conditions were as follows: $3 \mathrm{~min}$ at $94^{\circ} \mathrm{C}$, followed by 35 cycles of $30 \mathrm{~s}$ at $94^{\circ} \mathrm{C}, 30 \mathrm{~s}$ at $60^{\circ} \mathrm{C}, 30 \mathrm{~s}$ at $72^{\circ} \mathrm{C}$, and an extra $7 \mathrm{~min}$ extension at $72^{\circ} \mathrm{C}$. In addition, PCR reactions were carried out with pig genomic DNA (as positive control) and without DNA (as negative control). The statistical analyses of the PCR results were performed with the IMpRH mapping tool accessible at http://rhdev.toulouse .inra.fr/. Two-point and multiple-point $\mathrm{RH}$ analyses were used for the identification of linkage groups using LOD score threshold of 5.0.

2.4. Heterozygous Pig Identification and Imprinting Assay. SNPs of CDKN1C and NAP1L4 genes were searched via alignment of Landrace and Rongchang cDNA sequences. Genotyping was performed for single-nucleotide polymorphisms (SNPs) in each gene using genomic DNA of the $23 \mathrm{~F} 1$ individuals. SNPs were detected by direct sequencing of PCR products using primer pairs IJF1/R1, IJF2/R2 (Table 1), for CDKN1C and NAP1L4, respectively.

The allelic expression pattern of each gene was analyzed by RT-PCR of total RNA from samples heterozygous for SNPs. PCR products by each of the primer pairs described above were then sequenced in both directions. To eliminate the possibility of genomic DNA contamination in the RTPCR, primers $\beta$-actinF/R spanning intron 3 of pig $\beta$-actin were designed based on porcine $\beta$-actin (AY550069).

2.5. Quantitative Real-Time PCR. Quantitative real-time PCR was performed with SYBR Premix Ex TaqTM II kit in a Bio-Rad iQ 5 machine (USA). Primers QF1/R1, QF2/R2 and QF3/R3 (Table 1) were used to amplify a 136, 128, and 


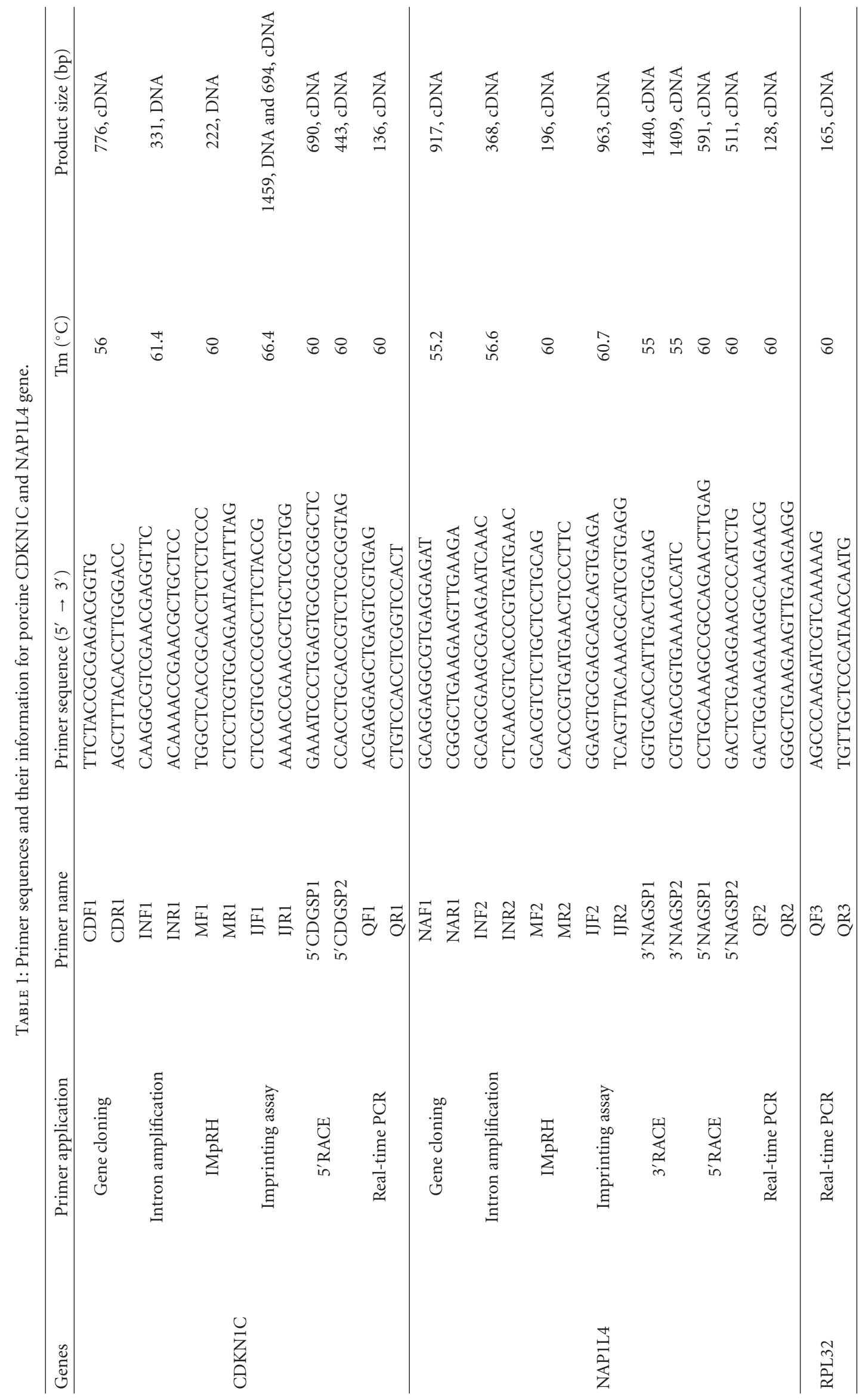


165 bp cDNA for CDKN1C, NAP1L4, and RPL32 (ribosomal protein L32), respectively. Melting curve analysis $\left(60-95^{\circ} \mathrm{C}\right)$ was used for assessing amplification specificity. Total RNA extracted from each tissue sample was reverse transcribed using PrimeScript RTase and oligo(dT) (TaKaRa, Japan). Reactions were performed in triplicate in a total volume of $25 \mu \mathrm{L}$. The reaction volume of $25 \mu \mathrm{L}$ contained $12.5 \mu \mathrm{L}$ SYBR Premix Ex Taq II (TaKaRa, Japan), $0.4 \mu \mathrm{M}$ each of primers, and $2.5 \mu \mathrm{L}$ template cDNA. A standard curve for analysis of the expression data was derived for each single gene from a serial dilution of the pool of cDNA samples. The endogenous control gene RPL32, which showed stable expression in our published data [24], was used. Relative expression of each gene was determined by using the $2^{-\Delta \Delta \mathrm{Ct}}$ method. ANOVA analysis and multiple comparisons were applied to each gene to verify whether the expression difference between tissues or ages was significant.

\section{Results}

3.1. Sequence Analysis and Localization of Porcine CDKN1C and NAP1L4. The full-length cDNA spanning $1171 \mathrm{bp}$ was assembled for porcine CDKN1C after sequencing the PCR products of primers CDF1/R1 as well as 5'-RACE (GenBank accession no. HQ679903). Sequence analysis showed that the gene contained an open reading frame (ORF) of $786 \mathrm{bp}$ (from nt 162 to nt 947 of HQ679903), encoding a 261amino acid protein. The ORF is $79 \%$ identical in amino acid sequence and $85 \%$ identical in nucleotide sequence to human sequences (NM_000076). The full-length cDNA of NAP1L4 is $2655 \mathrm{bp}$ (HQ679902) with $470 \mathrm{bp} \mathrm{5}$-UTR, $1024 \mathrm{bp} 3^{\prime}$ UTR, and $1161 \mathrm{bp}$ ORF, which codes for 386 amino acids. The porcine NAP1L4 utilizes a polyadenylation site (ATTAAA), located at position 2617 of HQ679902. The ORF is $94 \%$ identical in amino acid sequence and $86 \%$ identical in nucleotide sequence to human sequences (NM_005969).

In human and mouse, CDKN1C and NAP1L4 genes are closely linked [8]. The chromosomal location of porcine CDKN1C and NAP1L4 was determined by the IMpRH panel [23]. The two-point analysis showed that both CDKN1C (LOD 15.78, distance $0.15 \mathrm{cR}$, and retention frequency $16 \%$ ) and NAP1L4 (LOD 17.94, distance $0.11 \mathrm{cR}$, and retention frequency 19\%) were closely linked to IMpRH06175, on Sus scrofa chromosome 2 (SSC2). The multiple-point analysis demonstrated that CDKN1C was linked to 311840215 (upper marker, dist $0.34 \mathrm{cR}$ ) and IMpRH06175 (lower marker, dist $0.14 \mathrm{cR}$ ), while NAP1L4 was linked to 311840215 (upper marker, dist $0.36 \mathrm{cR}$ ) and IMpRH06175 (lower marker, dist $0.10 \mathrm{cR})$.

3.2. Expression and Imprinting of CDKN1C. The expression patterns of porcine CDKN1C were determined by relative quantitative real-time PCR analysis with total RNAs isolated from tissues of 5 neonatal and 7 one-month hybrids of $\mathrm{LD} \times$ RC. For each age, RNA of a tissue was used as a biological repeat. In neonatal pigs, the mRNA level of CDKN1C in placenta was amazingly high $(P<0.01)$, while it was very low in other tissues and with no differences between them

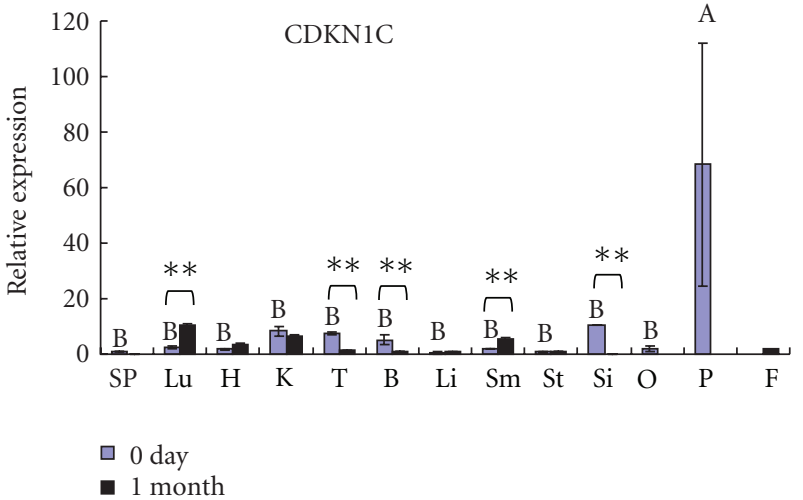

(a)

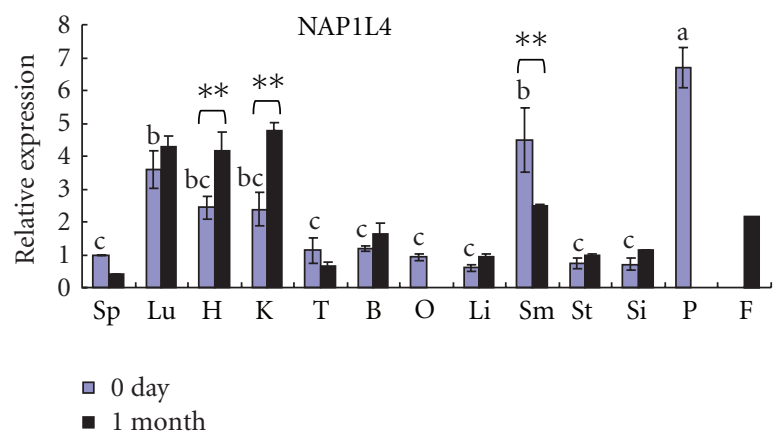

(b)

Figure 1: Tissue expression of porcine CDKN1C (a) and NAP1L4 (b). The expression values are normalized to RPL32 and expressed as mean \pm SE. Sp: spleen; Lu: lung; $\mathrm{H}$ : heart; K: kidney; T: tongue; B: bladder; O: ovary; Li: liver; Sm: skeletal muscle; St: stomach; Si: small intestine; P: placenta; F: fat. ABC-column means within 0 day of age bearing different superscripts differs significantly $P<0.05$ (lowercase) and $P<0.01$ (uppercase). ${ }^{* *} P<0.01$.

(Figure 1(a)). In one-month-old pigs, highest expression levels were observed in lung and kidney $(P<0.01)$, followed by skeletal muscle and heart $(P<0.01)$. Fewer CDKN1C transcripts were present in spleen, tongue, bladder, stomach, liver, fat, and small intestine $(P<0.01)$. Interestingly, transcript levels of porcine CDKN1C were upregulated in lung and skeletal muscle, and downregulated in tongue, bladder, and small intestine $(P<0.01)$, but no difference was seen in the other tissues after birth.

Alignment of Rongchang and Landrace sequences revealed an SNP (A/G) at position 916 of CDKN1C (HQ679903). Since the polymorphism did not provide any available RFLP site, direct sequencing of RT-PCR products was used for the discrimination of the expressed alleles. Sequencing of the PCR products revealed that three newborn pigs from $\mathrm{RC} \times$ $\mathrm{LD}$ and four from $\mathrm{LD} \times \mathrm{RC}$ occurred to be heterozygous for the SNP, while their parental pigs were homozygous for the allele $A$ and $G$, respectively. The $(\mathrm{RC} \times \mathrm{LD}) \mathrm{F} 1$ pigs only expressed the Landrace allele $\mathrm{G}$, and $(\mathrm{LD} \times \mathrm{RC}) \mathrm{F} 1$ pigs only expressed the Rongchang A allele in placenta (Figure 2(a)) as well as in tongue, bladder, ovary, spleen, liver, skeletal muscle, stomach, and small intestine. However, biallelic 

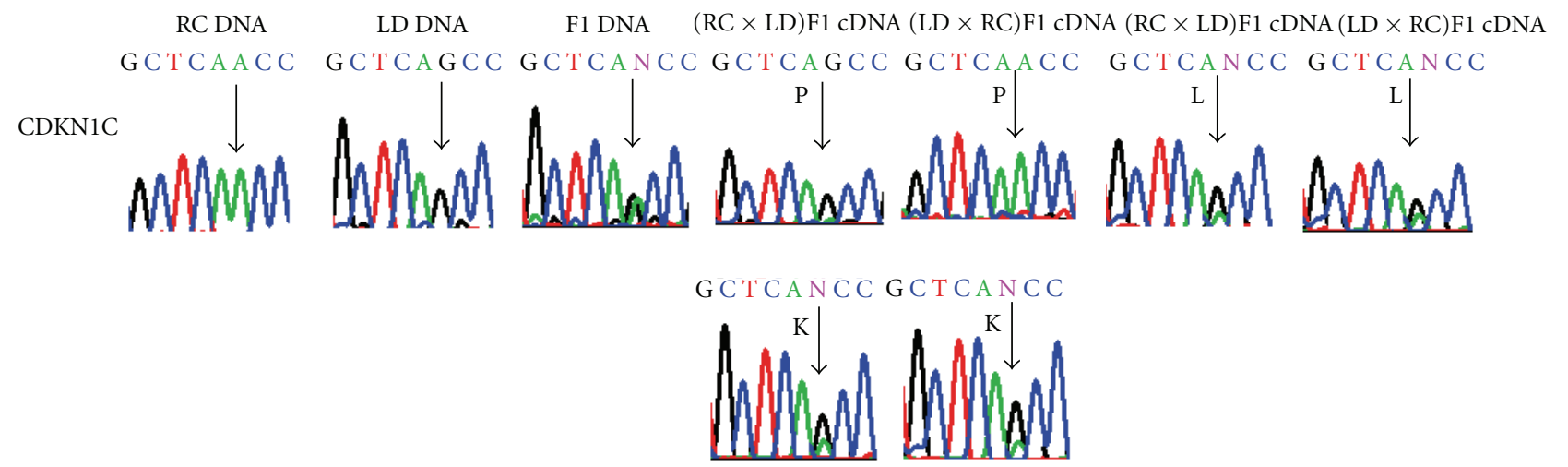

(a)

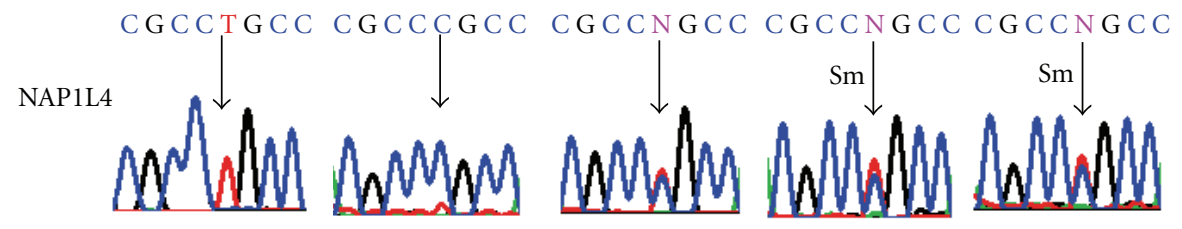

(b)

Figure 2: Maternal or biallelic expression of CDKN1C (a) and NAP1L4 (b) in 0-day pigs. RC: Rongchang pigs, LD: Landrace pigs, P: placenta, L: lung, K: kidney, Sm: skeletal muscle. Arrows indicate SNP sites.

expression of porcine CDKN1C was detected in lung and kidney (Figure 2(a)).

3.3. Expression and Imprinting of NAP1L4. NAP1L4 was ubiquitously expressed in porcine tissues. In the newborn pigs, the expression level was significantly higher in placenta $(P<0.01)$, followed by lung and skeletal muscle $(P<0.05)$. Fewer NAP1L4 transcripts were present in spleen, tongue, bladder, ovary, liver, stomach and small intestine $(P<0.05)$ (Figure 1(b)). In contrast, at the age of one month, highest levels of expression were observed in lung, heart, and kidney $(P<0.01)$ and the lowest expression were detected in spleen and tongue $(P<0.01)$. Compared with neonatal tissues, expression of porcine NAP1L4 was upregulated in heart and kidney, and downregulated in skeletal muscle $(P<0.01)$, but no significant changes were seen in the other tissues.

A T/C SNP was detected between Rongchang and Landrace pigs at position 2461 of NAP1L4 (HQ679902). A total of five DNA samples from $(\mathrm{RC} \times \mathrm{LD}) \mathrm{F} 1$ pigs and four DNA samples from $(\mathrm{LD} \times \mathrm{RC}) \mathrm{F} 1$ pigs were heterozygous for this polymorphism, while their parents Landrace and Rongchang pigs were $\mathrm{C} / \mathrm{C}$ and $\mathrm{T} / \mathrm{T}$ homozygotes, respectively. Direct sequencing of RT-PCR products demonstrated biallelic transcription of NAP1L4 in lung, heart, kidney, tongue, bladder, ovary, spleen, liver, skeletal muscle, stomach, small intestine, and spleen of newborn pigs. The biallelic expression of porcine NAP1L4 in skeletal muscle is illustrated in Figure 2(b).

\section{Discussion}

In this study, CDKN1C and NAP1L4 genes were cloned, and their chromosome location, expression patterns, and imprinting were further analyzed for the first time in pigs. We use Rongchang and Landrace breeds for analysis of allelic expression due to the fact that F1 hybrids of the two breeds are useful for detecting imprinting of genes [25]. Sequence analysis showed that porcine CDKN1C and NAP1L4 have high homology with those of human in amino acid and in nucleic acid, respectively, and both genes are closely linked in the region between markers IMpRH06175 and 311840215 on SSC2, syntenic to mice distal chromosome 7 and human 11 p15.5 [8]. These results indicate that both genes are conservative in mammals.

CDKN1C is a potent inhibitor of several G1 cyclin/CDK complexes, and its overexpression leads to cell-cycle arrest in the $\mathrm{G} 1$ phase $[26,27]$. The biological functions and distribution of CDKN1C in tissues have been widely investigated. In human and mouse, researches have shown that CDKN1C is mostly expressed in placenta and at low levels in other tissues, while it is expressed in adult heart, brain, lung, skeletal muscle, kidney, and pancreas [28, 29]. Recently, Shukla et al. [30] revealed that mRNA levels of CDKN1C were altered in placenta of intrauterine growth restriction (IUGR) and alcohol-consuming dams compared to the controls; thus, they were selected as the biomarkers for IUGR and intrauterine alcohol exposure. Mutations in mouse Cdkn1c result in the appearance of large thrombotic lesions in the labyrinth zone in mouse placenta [31]. Our work showed that the mRNA abundance of CDKN1C was highest in porcine neonatal placenta, while its high expression levels were detected in lung and kidney of one-month-old pigs. In general, the expression pattern of porcine CDKN1C correlates well with the human and mouse gene, and the high levels in expression of CDKN1C in porcine placenta illustrate 
its important roles in porcine placental growth and development.

NAP1L4 has been found to be localized mainly to the cytoplasm and a minor population of them to be in the nucleus, mediating nucleosome formation [32]. Hu et al. [33] found that NAP1L4 is ubiquitously expressed, showing a threefold higher level of expression in testis than in other tissues. Interestingly, our work also showed that NAP1L4 was ubiquitously expressed in porcine tissues, but higher expression of NAP1L4 was detected in neonatal placenta than in the other tissues. As NAP1L4 mediates angiogenesis, and it is significantly higher expressed in red-feather Taiwan country chickens of high egg productions than in the subpopulations of low egg productions [34]. These studies indicate that the functions of NAP1L4 contribute to reproduction performance in animals and may play a role in placental functions.

There are some reports about the imprinting of CDKN1C and NAP1L4. CDKN1C is expressed from maternal allele in both human and mouse [8]. Mouse Nap114 is paternally repressed in placenta and biallelically expressed in other embryo tissues [21], whereas biallelic expression of human NAP1L4 can be detected in all tissues and ages tested by Higashimoto et al. [8]. Like in humans, NAP1L4 in pigs was biallelically expressed in all tissues detected in this study. Furthermore, our data showed maternal expression of porcine CDKN1C in heart, tongue, bladder, ovary, spleen, liver, skeletal muscle, stomach, small intestine and placenta, consistent with previous data of greater expression of CDKN1C from parthenote than from biparental samples [22]. The biallelic expression of CDKN1C in lung and kidney in the pig differs from monoallelic expression in the same tissues in humans. This is because the degree of imprinting is often tissue and species specific because of different epigenetic modification [35]. In humans and mice, it has been documented that CDKN1C imprinting is regulated by KCNQ1OT1 antisenseRNA transcript originating from intron 10 of the maternally expressed KCNQ1 (KvLQT1) gene [36]. A CpG island located at the promoter of KCNQ1OT1, referred to as KvDMR1 or KCNQ1 imprinting control region (ICR), is unmethylated on the paternal allele and methylated on the maternal allele, favouring expression of KCNQ1OT1 from the paternal allele that leads to the propagation of silencing of neighboring genes including CDKN1C. While the KCNQ1 ICR has not been characterized in pigs, gene order and gene structure within this cluster are highly conservative between human, mouse, chicken, and tammar [37]. This information lends support to the hypothesis that different epigenetic modification of KCNQ1 ICR or other mechanisms terminatie the KCNQ1OT1 transcripts in porcine lung and kidney. Further studies are needed to elucidate the mechanisms associated with biallelic expression of CDKN1C in lung and kidney of pigs.

\section{Conclusions}

The full-length cDNA of porcine NAP1L4 and cDNA including $5^{\prime}$-UTR and ORF of porcine CDKN1C was cloned in this study. Both porcine CDKN1C and NAP1L4 were mapped on SSC2, closely linked to IMpRH06175. Imprinting analysis showed that NAP1L4 was biallelically expressed in the tissues tested, while CDKN1C was maternally expressed in tissues except lung and kidney in pigs, confirming the results by Bischoff et al. [22] that CDKN1C is expected of maternal expression. This study revealed a novel imprinted domain in the pig and provided data for further investigation of functional importance of porcine CDKN1C and NAP1L4.

\section{Acknowledgments}

The authors thank Dr. Martine Yerle and Denis Milan (INRA, Castanet-Tolosan, France) for kindly providing DNA of the IMpRH panel. This study was supported by the Natural Science Foundation Project of CQ CSTC (no. 2009BA1052) and The Fundamental Research Funds for Centre Universities (no. XDJK2009C153).

\section{References}

[1] S. Abu-Amero, D. Monk, J. Frost, M. Preece, P. Stanier, and G. E. Moore, "The genetic aetiology of Silver-Russell syndrome," Journal of Medical Genetics, vol. 45, no. 4, pp. 193-199, 2008.

[2] M. Charalambous, S. T. Da Rocha, and A. C. Ferguson-Smith, "Genomic imprinting, growth control and the allocation of nutritional resources: consequences for postnatal life," Current Opinion in Endocrinology, Diabetes and Obesity, vol. 14, no. 1, pp. 3-12, 2007.

[3] J. G. Falls, D. J. Pulford, A. A. Wylie, and R. L. Jirtle, "Genomic imprinting: implications for human disease," American Journal of Pathology, vol. 154, no. 3, pp. 635-647, 1999.

[4] R. D. Nicholls, "The impact of genomic imprinting for neurobehavioral and developmental disorders," Journal of Clinical Investigation, vol. 105, no. 4, pp. 413-418, 2000.

[5] P. P. Luedi, A. J. Hartemink, and R. L. Jirtle, "Genome-wide prediction of imprinted murine genes," Genome Research, vol. 15, no. 6, pp. 875-884, 2005.

[6] I. Nikaido, C. Saito, Y. Mizuno et al., "Discovery of imprinted transcripts in the mouse transcriptome using large-scale expression profiling," Genome Research, vol. 13, no. 6, pp. 14021409, 2003.

[7] W. Reik and J. Walter, "Genomic imprinting: parental influence on the genome," Nature Reviews Genetics, vol. 2, no. 1, pp. 21-32, 2001.

[8] K. Higashimoto, H. Soejima, T. Saito, K. Okumura, and T. Mukai, "Imprinting disruption of the CDKN1C/KCNQ1OT1 domain: the molecular mechanisms causing Beckwith-Wiedemann syndrome and cancer," Cytogenetic and Genome Research, vol. 113, no. 1-4, pp. 306-312, 2006.

[9] M. H. Lee, I. Reynisdottir, and J. Massague, "Cloning of p57(KIP2), a cyclin-dependent kinase inhibitor with unique domain structure and tissue distribution," Genes and Development, vol. 9, no. 6, pp. 639-649, 1995.

[10] H. Watanabe, Z. Q. Pan, N. Schreiber-Agus, R. A. DePinho, J. Hurwitz, and Y. Xiong, "Suppression of cell transformation by the cyclin-dependent kinase inhibitor pSTKIP2 requires binding to proliferating cell nuclear antigen," Proceedings of the National Academy of Sciences of the United States of America, vol. 95, no. 4, pp. 1392-1397, 1998.

[11] I. Hatada, A. Nabetani, H. Morisaki et al., "New p57 ${ }^{\mathrm{KIP} 2}$ mutations in Beckwith-Wiedemann syndrome," Human Genetics, vol. 100, no. 5-6, pp. 681-683, 1997. 
[12] W. W. K. Lam, I. Hatada, S. Ohishi et al., "Analysis of germline CDKNIC $\mathrm{p} 57^{\mathrm{KIP} 2}$ mutations in familial and sporadic Beckwith-Wiedemann syndrome (BWS) provides a novel genotype-phenotype correlation," Journal of Medical Genetics, vol. 36, no. 7, pp. 518-523, 1999.

[13] P. Zhang, N. J. Liégeois, C. Wong et al., "Altered cell differentiation and proliferation in mice lacking p57(KIP2) indicates a role in Beckwith-Wiedemann syndrome," Nature, vol. 387, no. 6629, pp. 151-158, 1997.

[14] T. Kikuchi, M. Toyota, F. Itoh et al., "Inactivation of p57KIP2 by regional promoter hypermethylation and histone deacetylation in human tumors," Oncogene, vol. 21, no. 17, pp. 27412749, 2002.

[15] Y. Li, H. Nagai, T. Ohno et al., "Aberrant DNA methylation of $\mathrm{p} 57^{\mathrm{KIP} 2}$ gene in the promoter region in lymphoid malignancies of B-cell phenotype," Blood, vol. 100, no. 7, pp. 2572-2577, 2002.

[16] L. L. Shen, M. Toyota, Y. Kondo et al., "Aberrant DNA methylation of $\mathrm{p} 57^{\mathrm{KIP} 2}$ identifies a cell-cycle regulatory pathway with prognostic impact in adult acute lymphocytic leukemia," Blood, vol. 101, no. 10, pp. 4131-4136, 2003.

[17] H. Soejima, T. Nakagawachi, W. Zhao et al., "Silencing of imprinted CDKN1C gene expression is associated with loss of CpG and histone H3 lysine 9 methylation at DMR-LIT1 in esophageal cancer," Oncogene, vol. 23, no. 25, pp. 4380-4388, 2004.

[18] M. J. Hoffmann, A. R. Florl, H. H. Seifert, and W. A. Schulz, "Multiple mechanisms downregulate CDKN1C in human bladder cancer," International Journal of Cancer, vol. 114, no. 3, pp. 406-413, 2005.

[19] P. Rodriguez, D. Munroe, D. Prawitt et al., "Functional characterization of human nucleosome assembly protein-2 (NAP1L4) suggests a role as a histone chaperone," Genomics, vol. 44, no. 3, pp. 253-265, 1997.

[20] P. Zhang, C. Wong, R. A. DePinho, J. W. Harper, and S. J. Elledge, "Cooperation between the Cdk inhibitors p27(KIP1) and p57(KRP2) in the control of tissue growth and development," Genes and Development, vol. 12, no. 20, pp. 3162-3167, 1998.

[21] D. Umlauf, Y. Goto, R. Cao et al., "Imprinting along the Kcnq1 domain on mouse chromosome 7 involves repressive histone methylation and recruitment of Polycomb group complexes," Nature Genetics, vol. 36, no. 12, pp. 1296-1300, 2004.

[22] S. R. Bischoff, S. Tsai, N. Hardison et al., "Characterization of conserved and nonconserved imprinted genes in swine," Biology of Reproduction, vol. 81, no. 5, pp. 906-920, 2009.

[23] M. Yerle, P. Pinton, A. Robic et al., "Construction of a wholegenome radiation hybrid panel for high-resolution gene mapping in pigs," Cytogenetics and Cell Genetics, vol. 82, no. 3-4, pp. 182-188, 1998.

[24] C. Jiang, P. Shi, S. Li et al., "Gene expression profiling of skeletal muscle of nursing piglets," International Journal of Biological Sciences, vol. 6, no. 7, pp. 627-638, 2010.

[25] C. D. Jiang, S. Li, and C. Y. Deng, "Assessment of genomic imprinting of PPP1R9A, NAP1L5 and PEG3 in pigs," Russian Journal of Genetics, vol. 47, no. 4, pp. 471-476, 2011.

[26] A. Tsugu, K. Sakai, P. B. Dirks et al., "Expression of p57KIP2 potently blocks the growth of human astrocytomas and induces cell senescence," American Journal of Pathology, vol. 157, no. 3, pp. 919-932, 2000.

[27] S. Matsuoka, M. C. Edwards, C. Bai et al., "p57 ${ }^{\mathrm{KIP} 2}$, a structurally distinct member of the $\mathrm{p} 21^{\mathrm{CIP} 1} \mathrm{Cdk}$ inhibitor family, is a candidate tumor suppressor gene," Genes and Development, vol. 9, no. 6, pp. 650-662, 1995.
[28] M. H. Lee, I. Reynisdottir, and J. Massague, "Cloning of p57(KIP2), a cyclin-dependent kinase inhibitor with unique domain structure and tissue distribution," Genes and Development, vol. 9, no. 6, pp. 639-649, 1995.

[29] J. Madhavan, K. Mallikarjuna, K. Vikas, R. George, R. Bremner, and G. Kumaramanickavel, "CDKN1C p57 KIP2 mRNA expression in human retinoblastomas," Ophthalmic Genetics, vol. 31, no. 3, pp. 141-146, 2010.

[30] P. K. Shukla, L. J. Sittig, T. M. Ullmann, and E. E. Redei, "Candidate placental biomarkers for intrauterine alcohol exposure," Alcoholism, vol. 35, no. 3, pp. 559-565, 2011.

[31] S. J. Tunster, M. Van De Pette, and R. M. John, "Fetal overgrowth in the Cdkn1c mouse model of Beckwith-Wiedemann syndrome," DMM Disease Models and Mechanisms, vol. 4, no. 6, pp. 814-821, 2011.

[32] M. Okuwaki, K. Kato, and K. Nagata, "Functional characterization of human nucleosome assembly protein 1-like proteins as histone chaperones," Genes to Cells, vol. 15, no. 1, pp. 13-27, 2010.

[33] R. J. Hu, M. P. Lee, L. A. Johnson, and A. P. Feinberg, "A novel human homologue of yeast nucleosome assembly protein, 65 $\mathrm{kb}$ centromeric to the $\mathrm{p} 57$ (KIP2) gene, is biallelically expressed in fetal and adult tissues," Human Molecular Genetics, vol. 5, no. 11, pp. 1743-1748, 1996.

[34] L. R. Chen, C. H. Chao, C. F. Chen, Y. P. Lee, Y. L. Chen, and Y. L. Shiue, "Expression of 25 high egg production related transcripts that identified from hypothalamus and pituitary gland in red-feather Taiwan country chickens," Animal Reproduction Science, vol. 100, no. 1-2, pp. 172-185, 2007.

[35] J. C. Y. Lau, M. L. Hanel, and R. Wevrick, "Tissue-specific and imprinted epigenetic modifications of the human NDN gene," Nucleic Acids Research, vol. 32, no. 11, pp. 3376-3382, 2004.

[36] D. Mancini-DiNardo, S. J. S. Steele, J. M. Levorse, R. S. Ingram, and S. M. Tilghman, "Elongation of the Kcnqlot1 transcript is required for genomic imprinting of neighboring genes," Genes and Development, vol. 20, no. 10, pp. 1268-1282, 2006.

[37] E. I. Ager, A. J. Pask, H. M. Gehring, G. Shaw, and M. B. Renfree, "Evolution of the CDKN1C-KCNQ1 imprinted domain," BMC Evolutionary Biology, vol. 8, no. 1, article no. 163, 2008. 

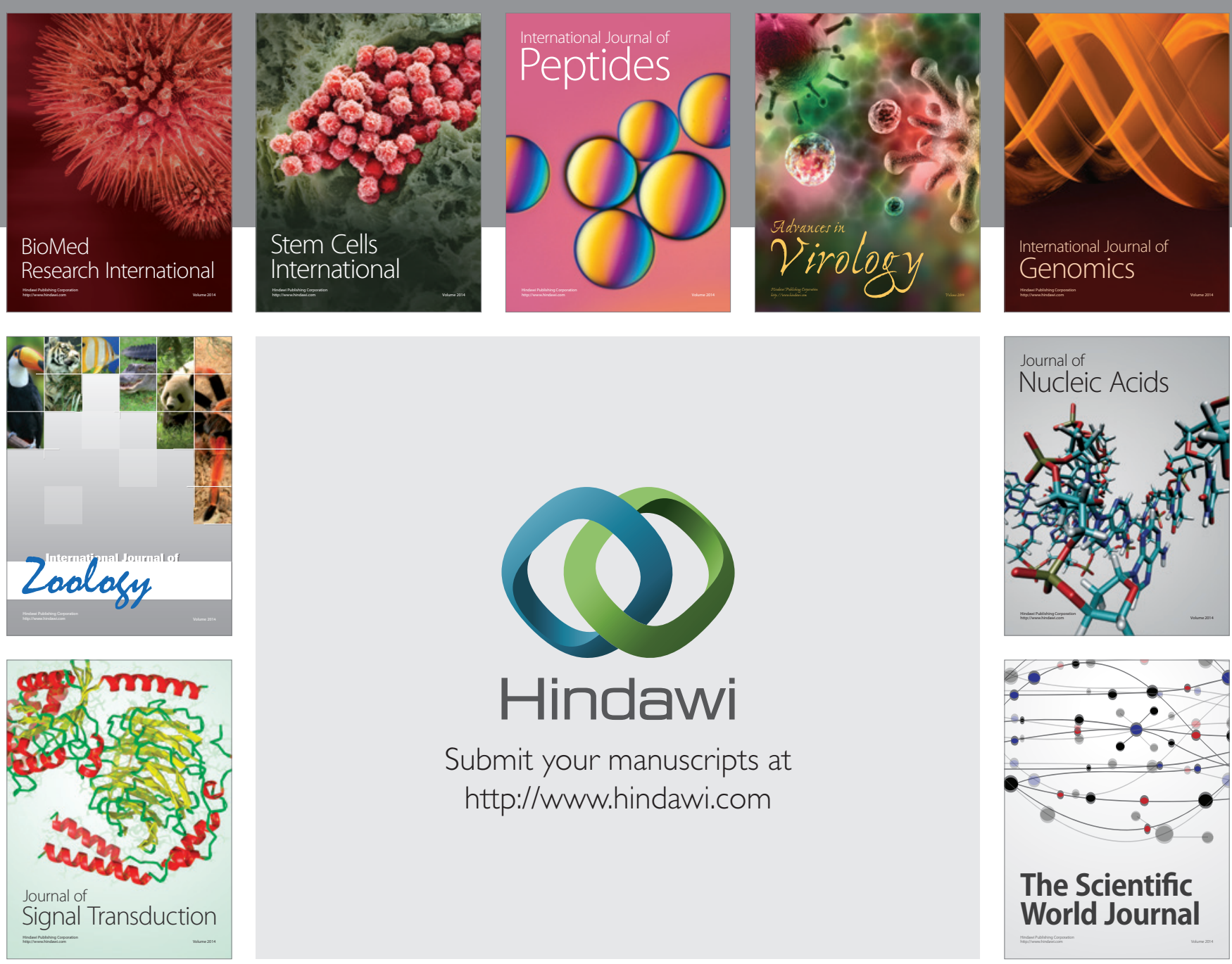

Submit your manuscripts at

http://www.hindawi.com
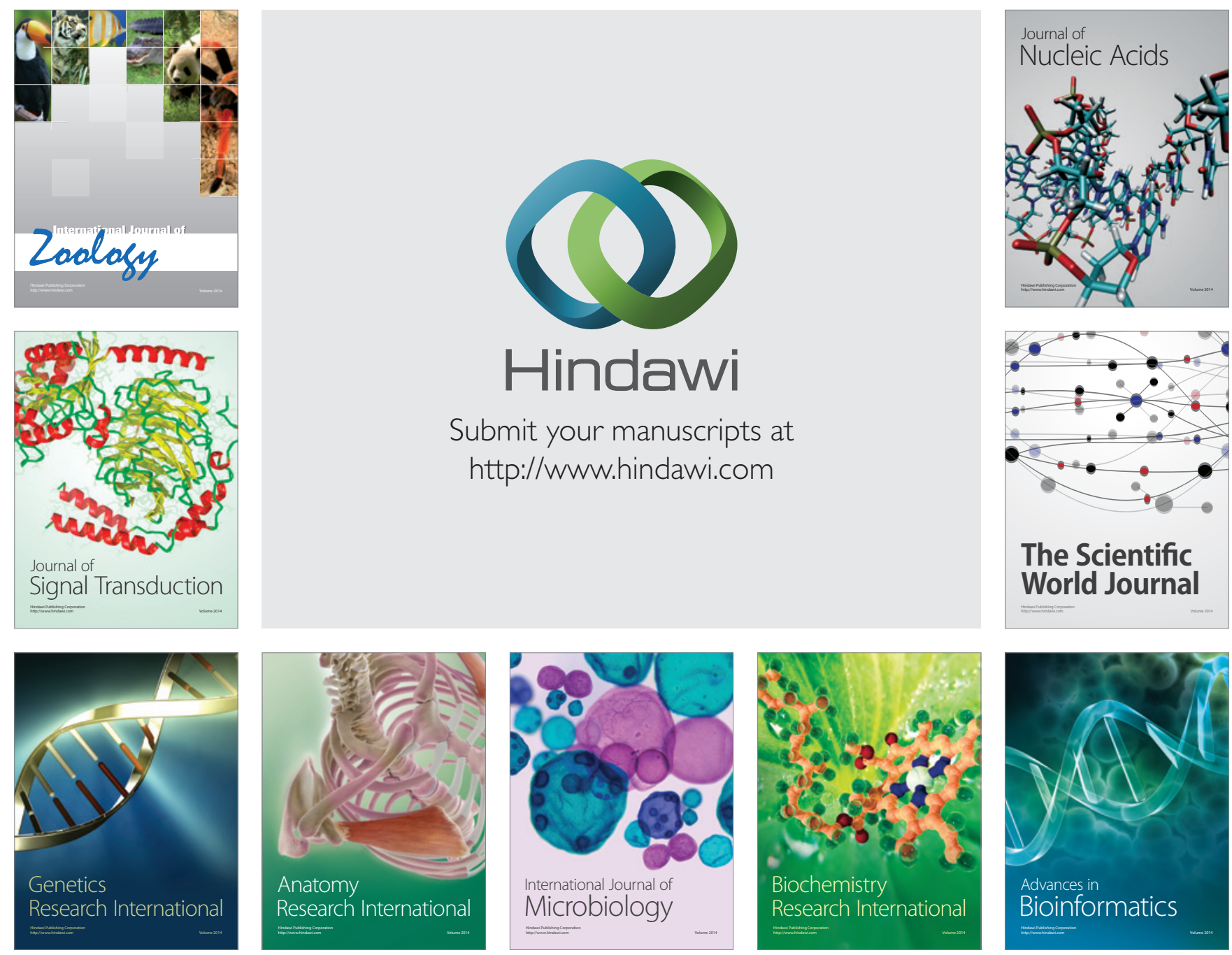

The Scientific World Journal
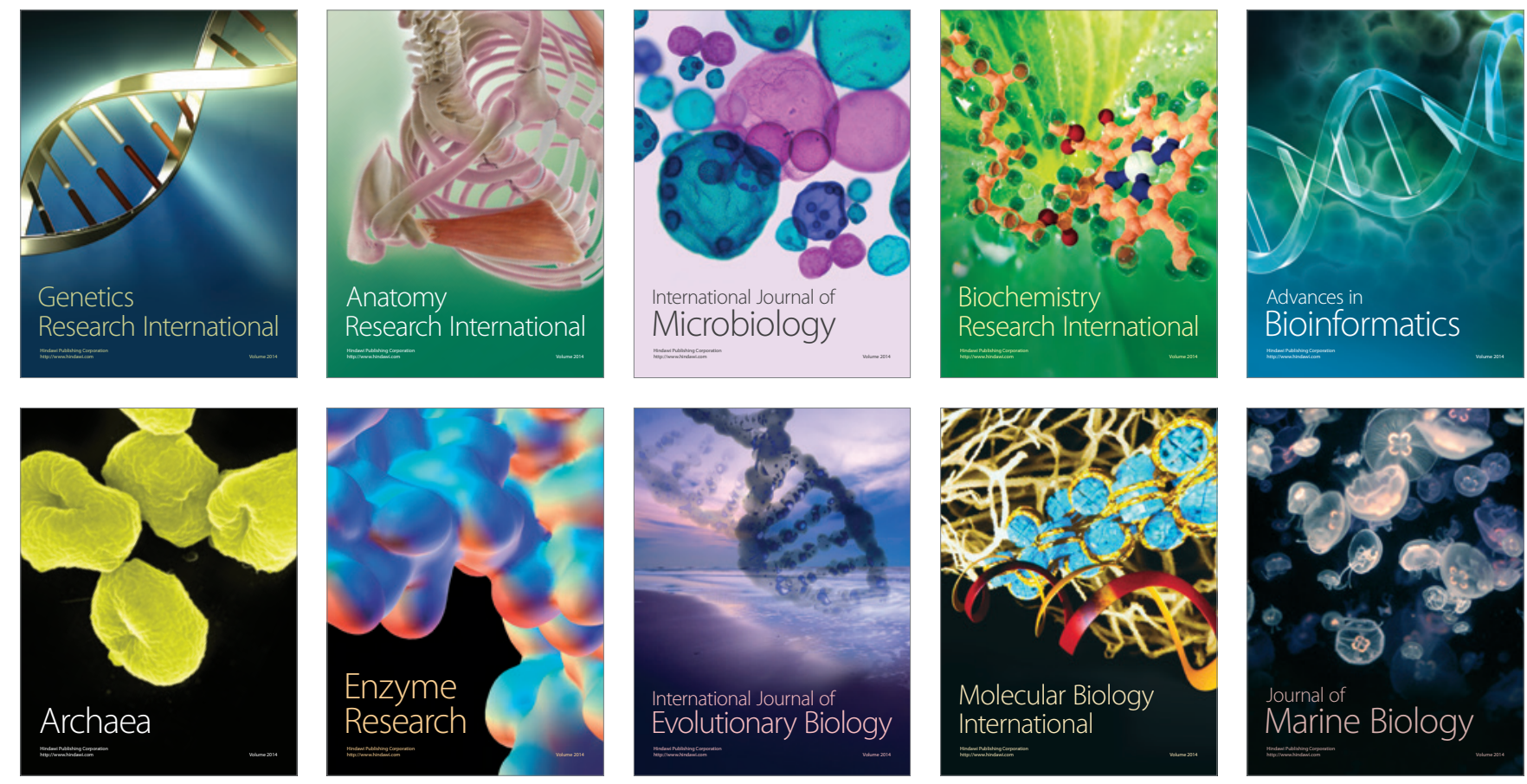